\title{
Back-drivable and Inherently Safe Mechanism for Artificial Finger
}

\author{
Koichi Koganezawa
}

\begin{abstract}
This paper shows mechanisms for artificial finger based on a planetary gear system (PGS). Using the PGS as a transmitter provides an under-actuated system for driving three joints of a finger with back-drivability that is crucial characteristics for fingers as an end-effector when it interacts with external environment. This paper also shows the artificial finger employed with the originally developed mechanism called "double planetary gear system" (DPGS). The DPGS provides not only back-drivable and under-actuated flexion-extension of the three joints of a finger, which is identical to the former, but also adduction-abduction of the MP joint. Both of the above finger mechanisms are inherently safe due to being back-drivable with no electric device or sensor in the finger part. They are also rigorously solvable in kinematics and kinetics as shown in this paper.
\end{abstract}

\section{INTRODUCTION}

Artificial finger has been strenuously studied in recent two decades since an anthropomorphic robot or spacecraft manipulator requires end-effectors to achieve dexterous task. However it is tremendously difficult to develop a hand having multiple fingers with a lot of DOF, which is feasible and practical not only on a mechanical point of view but also on their control. Some studies have been pursued to produce a hand of which fingers have full of DOF; each one is controlled by tiny DC-motor assembled in the finger [1][2] or by a remote control via wires[3][4][5]. This approach is interested in developing a research platform to study various control methods of multi-DOF hand when it handles objects, but it may not be oriented for practical usage as a hand for anthropomorphic robots or forearm prostheses.

In the meanwhile practical usage for robot hands or forearm prostheses requires reducing DOF. There have been some studies to control three joints of one finger (MP, PIP, DIP joints) by one motor via pulleys and wire [6] [7] or closed linkage system. It is a matter of course that the motion of the finger developed in these studies is restricted in one typical motion such as open-and-grip.

The study of this paper aims to develop finger mechanisms and a hand with multiple-fingers that can achieve dexterous handling tasks while sustaining feasibility for practical usage. The author considers an artificial finger or hand should have following properties to take into consideration for practical usage.

Manuscript received February 1, 2010. This work was supported in part by Japan Society for the Promotion of Science, via Grants-in-Aid for Scientific Research: No. 20560242 'Development of Artificial Hand using the Double Planetary Gear System and the Handling of Objects'.

Koichi Koganezawa is the Professor of the Department of Mechanical Engineering, Tokai University (e-mail: kogane@keyaki.cc.u-tokai.ac.jp)
Back-drivability (BD): The hand with multiple fingers is an end-effecter that always interacts with external environment, of which effect to the fingers is usually not expected to be predictable. This fact requires a hand to have some "resiliency" to the external interaction, which is interpreted as "back-drivability" of the joints of the hand in the technical point of view. This property have been pursued in some studies to develop tendon-driven hand [6][7] .

Inherently safe design (ISD): This property is much related to the $\mathrm{BD}$ in the meaning that the hand never harmful to external environment (especially to human). In addition to the $\mathrm{BD}$, ISD requires some items to a controller of the fingers as follows.

(a) Any electric/electronic devices or sensors should not be equipped in the finger part. Because of the trait of the fingers as an end-effecter, it has to cope with severe external environments to be possible, such as electromagnetic or radioactive fields.

(b) According to (a) the controller can not much rely on the feedback from sensors such as tactile or pressure sensors.

ISD does not completely deny using the sensory feedback, which might be helpful to accomplish some dexterous and precise motion of the hand if a measure against noise is satisfactory. Therefore ISD requires that some primitive motions of the fingers should be provided without sensory feedback, in other words, mechanically, but it allows using the sensory feedback as subsidiary measures.

All-in-one design (AOD): All of the actuators should be mounted in a hand (distal to the wrist). A lot of works have equipped the actuators at the place away from the hand [3][4][5], the forearm part for example, and taken the wire driven as a force/torque transmission. But it will hamper to compose a wrist joint that has three DOF if trying to mimic a human. Moreover the wire-driven have some drawbacks; friction loss, control delay, unpredictable non-linear behavior due to the friction and the elastic element of the wire, all of which make the control precision worse. The place for mounting the actuator is therefore severely restricted due to AOD and ISD requirements. Only the palm is remained for it. Therefore the AOD requires reducing the number of actuators as many as possible by employing some under-actuation mechanical systems.

Simple Control (SC): The control of the multiple fingers of the hand should be as simple as possible. This requirement will be achieved along with pursuing the above BD and ISD properties. BD will reduce the control burden to determine the fingers' shape when they interact with external objects. ISD 
also provides the simple control that does not much rely on the sensory feedback.

Hence the purpose of the study is to develop a finger and a hand with multiple fingers that meet or have a potential to meet the above properties, BD, ISD, AOD and SC.

This paper is organized as follow. In the following section it shows a finger using a planetary gear system, the principle of its motion, shape-fitting property, kinematics and kinetics to show the back-drivability. In the third section it shows the advanced finger mechanism using the double planetary gear system. The last section is devoted to the conclusive remarks.

\section{Finger Using Planetary Gear System[8]}

\section{A. Structure}

Fig. 1 shows the structure of the finger. It has three joint (DIP, PIP and MP joints) that are connected with a compound fourbar linkage system and a planetary gear system. The link 1 is directly attached to the inner gear to rotate with it. The link 2 is connected to one of the planetary gear axis. The size is almost the same as an adult index finger. The compound four-bar

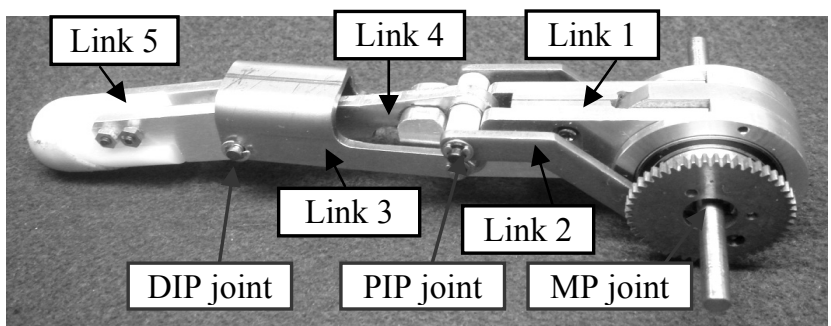

(a) Whole appearance of the finger

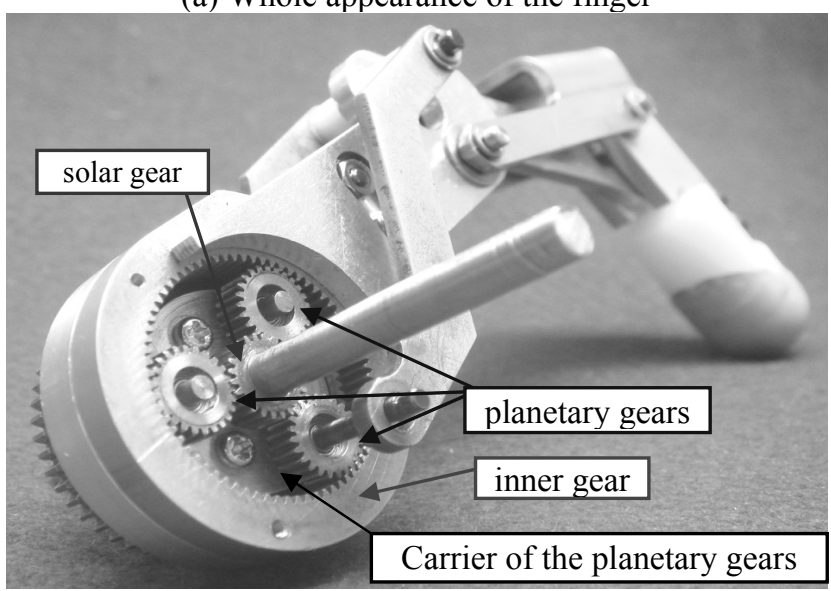

(b) Planetary gear system ay the MP joint

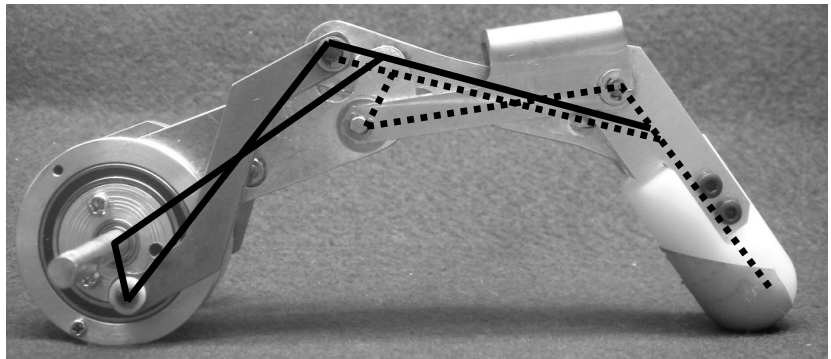

(c) Compound four-bar linkages

Fig. 1 The finger using the planetary gear system
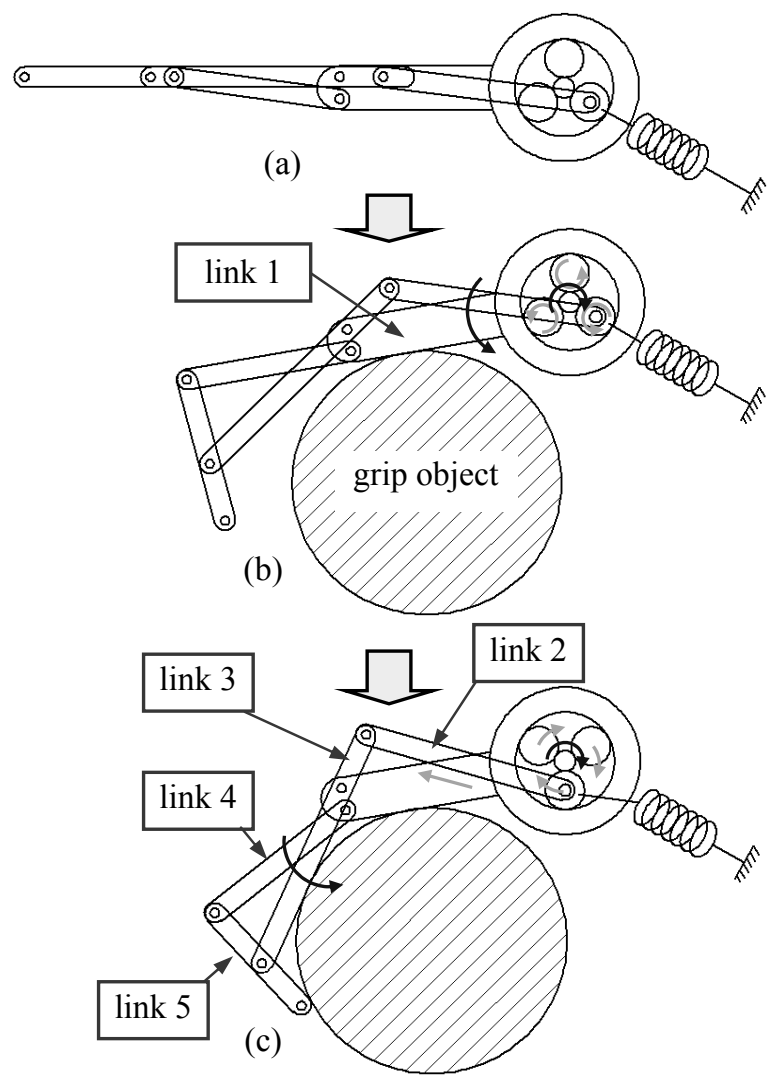

Fig.2 Sequence of the synergetic gripping motion

linkage system developed in this study is in part similar to the parallel mechanism developed in Laval University[9]. However our mechanism can be achieved by lesser number of parts in a smaller volume.

\section{B. Synergetic gripping motion}

The new mechanism allows the three joints of one finger (DIP, PIP and MP joints) to synergically wrap an object with no sensory feedback.

The key point of the mechanism resides in the fact that the planetary gear system is a transmitter that receives two inputs; the solar gear receives active torque from an actuator, while the carrier of the planetary gear receives a passive torque from some elastic element or some dissipative element. This amalgamation of active and passive torques provides synergetic motion of the three joints according to the shape of a gripping object under a simple control.

Fig.2 illustrates the motion sequence of gripping an object. The spring pulls the carrier of the planetary gears of which force is strong enough to sustain the weight of the finger and not to have the carrier rotate under no external force lording to the finger (a).

The clock-wise rotation of the solar gear gives rise to the counter clock-wise rotation of the inner gear via the rotation of the planetary gears on their axes (b).

Once the motion of the link 1 is hampered by touching the object and the rotation of the inner gear is stopped, further clock-wise rotation of the solar gears give rise to the orbital 


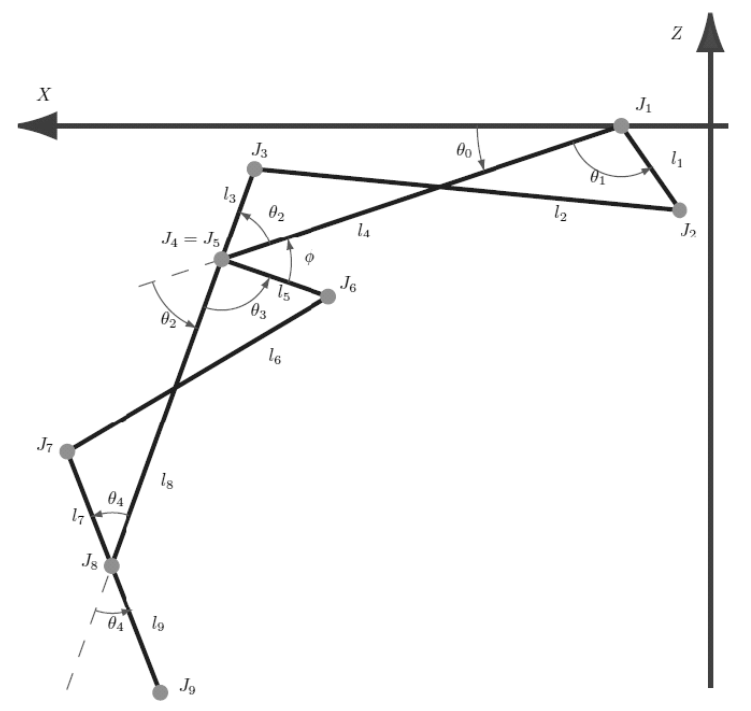

Fig.3 Finger configuration

rotation of the planetary gears around the solar gear accompanied by the rotation of the carrier. The rotation of the carrier push the link 2 forward, which leads the wrapping motion of the object by the link 3, 4 and 5 (c).

It is noteworthy that the line of motions described above can be carried out only by one-way rotation of the solar gears with no sensory feedback. Therefore this mechanism will meet the entire requirement of $\mathrm{BD}, \mathrm{ISD}, \mathrm{AOD}$ and $\mathrm{SC}$ defined in the former section.

\section{Kinematics and kinetics}

According to the assignment of the joint angles and the links shown in Fig.3, we can derive the kinematics and the kinetics of the finger. First we find that the angles $\theta_{0}$ and $\theta_{1}$ are related to the solar gear angle $\theta_{S}$ and the carrier angle $\theta_{P}$ described by,

$$
\left[\begin{array}{c}
\theta_{0} \\
\theta_{1}
\end{array}\right]=\left[\begin{array}{cc}
-Z_{S} / Z_{I} & \left(Z_{S}+Z_{I}\right) / Z_{I} \\
Z_{S} / Z_{I} & -Z_{S} / Z_{I}
\end{array}\right]\left[\begin{array}{c}
\theta_{S} \\
\theta_{P}
\end{array}\right]=\mathbf{A}\left[\begin{array}{c}
\theta_{S} \\
\theta_{P}
\end{array}\right]
$$

where, $Z_{S}$ and $Z_{I}$ are the number of teeth of the solar gear and the inner gear respectively.

Next we can derive the two constraint equations for determining the dependent variables $\theta_{2}$ and $\theta_{4}\left(\theta_{3}\right.$ is equal to $\pi-\phi-\theta_{2}$ with the constant angle $\phi$ ) as,

$$
\boldsymbol{\Phi}=\left[\begin{array}{l}
\left(\mathbf{J}_{\mathbf{2}}-\mathbf{J}_{\mathbf{3}}\right)^{T}\left(\mathbf{J}_{\mathbf{2}}-\mathbf{J}_{\mathbf{3}}\right)-l_{2}^{2} \\
\left(\mathbf{J}_{\mathbf{6}}-\mathbf{J}_{\mathbf{7}}\right)^{T}\left(\mathbf{J}_{\mathbf{6}}-\mathbf{J}_{\mathbf{7}}\right)-l_{6}^{2}
\end{array}\right]=\mathbf{0}_{2}
$$

Partial differentiation of (2) with respect to $\theta_{0} \theta_{1}, \theta_{2}$ and $\theta_{4}$ provides,

$$
\mathbf{B}\left[\begin{array}{cc}
\Delta \theta_{2} & \Delta \theta_{4}
\end{array}\right]^{T}+\mathbf{C}\left[\begin{array}{ll}
\Delta \theta_{0} & \Delta \theta_{1}
\end{array}\right]^{T}=\mathbf{0}_{2}
$$

with

$$
\mathbf{B}=\left[\begin{array}{ll}
\partial \boldsymbol{\Phi}_{i} / \partial \theta_{2} & \partial \boldsymbol{\Phi}_{i} / \partial \theta_{4}
\end{array}\right], \quad \mathbf{C}=\left[\begin{array}{ll}
\partial \boldsymbol{\Phi}_{i} / \partial \theta_{0} & \partial \boldsymbol{\Phi}_{i} / \partial \theta_{1}
\end{array}\right]
$$

It follows by combining (3) with (1),

$$
\left[\begin{array}{ll}
\Delta \theta_{2} & \Delta \theta_{4}
\end{array}\right]^{T}=-\mathbf{B}^{-1} \mathbf{C A}\left[\begin{array}{ll}
\Delta \theta_{S} & \Delta \theta_{P}
\end{array}\right]^{T}
$$

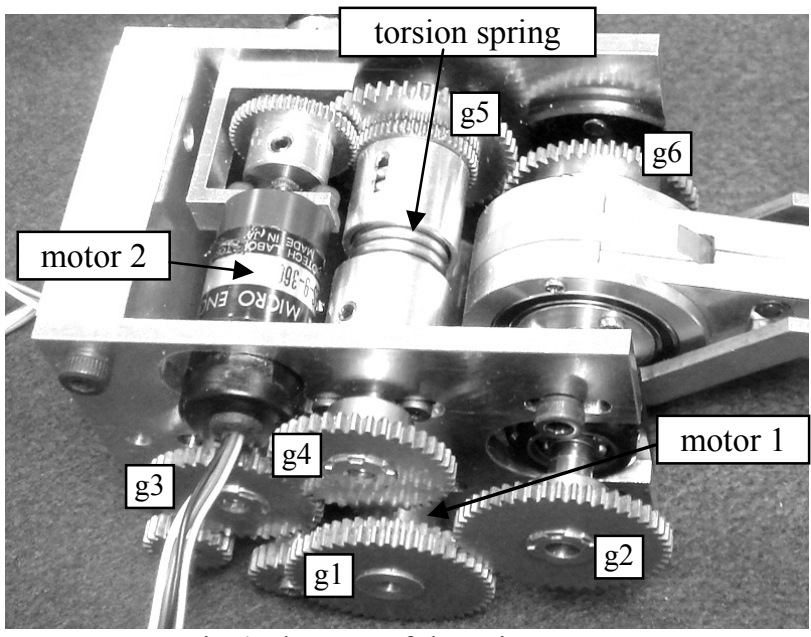

Fig.4 The part of the prime mover

By using (1) and (4) we can determine all of the dependent angles from the infinitesimal variations of $\theta_{S}$ and $\theta_{P}$.

The infinitesimal variation of the finger tip position $\mathbf{J}_{9}=\left[\begin{array}{lll}J_{9 z} & J_{9 x}\end{array}\right]^{T}$ is calculated by,

with

$$
\left[\begin{array}{ll}
\Delta J_{9 z} & \Delta J_{9 x}
\end{array}\right]^{T}=\mathbf{D}\left[\begin{array}{ll}
\Delta \theta_{2} & \Delta \theta_{4}
\end{array}\right]^{T}+\mathbf{E}\left[\begin{array}{ll}
\Delta \theta_{0} & \Delta \theta_{1}
\end{array}\right]^{T}
$$

$$
\mathbf{D}=\left[\begin{array}{ll}
\partial \mathbf{J}_{9} / \partial \theta_{2} & \partial \mathbf{J}_{9} / \partial \theta_{4}
\end{array}\right], \quad \mathbf{E}=\left[\begin{array}{ll}
\partial \mathbf{J}_{9} / \partial \theta_{0} & \partial \mathbf{J}_{9} / \partial \theta_{1}
\end{array}\right]
$$

It follows the final result by substituting (1) and (4) into (5),

$$
\left[\begin{array}{ll}
\Delta J_{9 z} & \Delta J_{9 x}
\end{array}\right]^{T}=\left(\mathbf{E}-\mathbf{D B} \mathbf{B}^{-1} \mathbf{C}\right) \mathbf{A}\left[\begin{array}{ll}
\Delta \theta_{S} & \Delta \theta_{P}
\end{array}\right]^{T}
$$

and we can identify the Jacobian of the finger,

$$
\boldsymbol{\Lambda}=\left(\mathbf{E}-\mathbf{D B}^{-1} \mathbf{C}\right) \mathbf{A}
$$

Once the Jacobian is identified we can establish the kinetics that relates the force at the finger tip $\left(J_{9}\right) \mathbf{f}=\left[\begin{array}{ll}f_{Z} & f_{X}\end{array}\right]^{T}$ and the torque of the solar gear and the carrier of the planetary gears $\tau$ $=\left[\begin{array}{ll}\tau_{S} & \tau_{P}\end{array}\right]^{T}$ as based on the virtual work principle,

$$
\boldsymbol{\tau}=\boldsymbol{\Lambda}^{T} \mathbf{f}
$$

\section{Control of the force of the finger tip}

The force exerted at the finger tip can be controlled if one extra motor is equipped to operate the spring tension. Fig. 4 shows the part of the prime mover of the finger. The torque transmission passes are as follows.

Motor $1 \rightarrow \mathrm{g} 1 \rightarrow \mathrm{g} 2 \rightarrow$ solar gear

Motor $2 \rightarrow \mathrm{g} 3 \rightarrow \mathrm{g} 4 \rightarrow$ torsion spring $\rightarrow \mathrm{g} 5 \rightarrow \mathrm{g} 6 \rightarrow$ carrier

The carrier is basically rotated passively accompanied by twisting the torsion spring. The force exerted at the finger tip is determined by a torque of the solar gear and a passive torque of the torsion spring. Therefore if the motor 2 adjusts the torsion angle of the spring, the force at the finger tip can be controlled according to the eq.(8).

\section{E. Pinching an object by two fingers}

The developed finger mechanism shown above allows us to scheme a very simple control when the two fingers pinch an object and handle it. Basically the motor 1 that directly drives the solar gear has a role to handle the object position, while the 


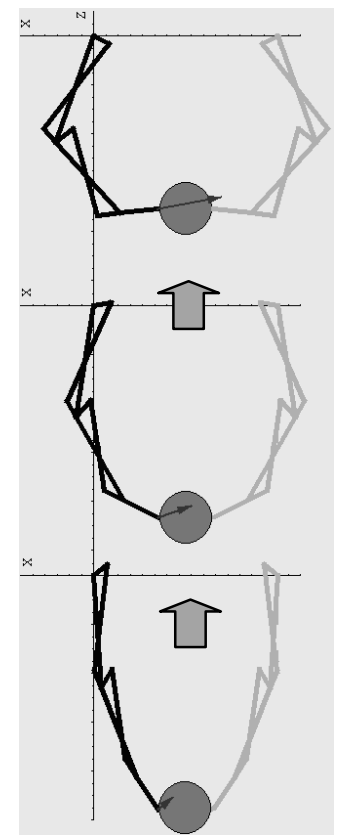

(a) No torsion spring control

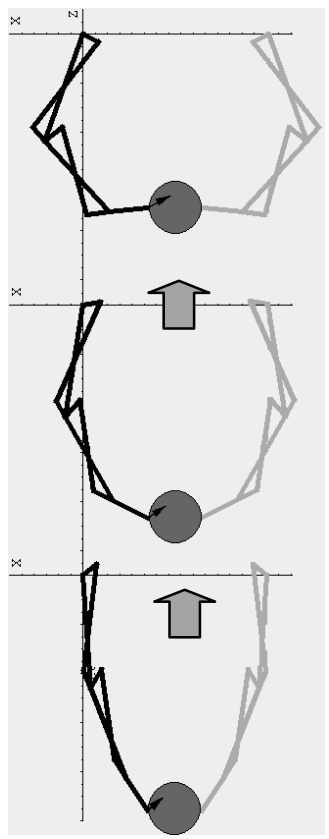

(b) Torsion spring control
Fig.5 Kinetic simulation of the object lifting-up by two fingers

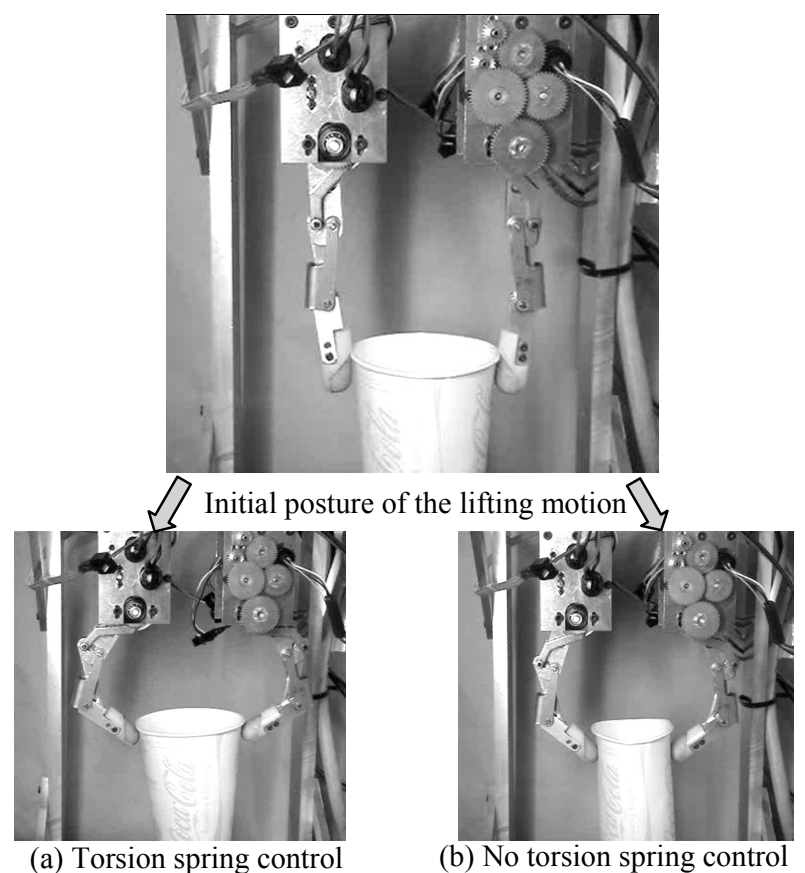

Fig.6 Lifting experiment by two fingers

motor 2 that twists the torsion spring has a role to adjust the force exerted at the finger tip, although they are not rigorously discriminated.

For example the simple lifting-up of an object by two fingers can be achieved by just controlling the motor 1. Fig.5 shows the kinetic simulation of lifting the $0.1 \mathrm{~kg}$ object. Fig.5 (a) is the results when the motor 1 exerts the necessary torque $\tau_{S}$ to hold the lifting posture and the torsion spring exerts the carrier torque $\tau_{P}$ that is passively determined by the rotation angle of the carrier. The arrows shown at the finger tip designate the force vector (only those of the left finger are shown). As shown, the force exerted at the finger tip increases according to the lifting height because the torsion spring is more twisted as proceeding the lifting. Fig.5 (a), on the other hand, is the result that adds the torsion spring control, in which the motor 2 controls the torsion angle of the spring to be always constant. As shown in the force vector at the finger tip, the pinching force is sustained to be almost constant during the lifting. Fig. 6 is the experimental result, of which control method is identical to the simulation. It shows the paper cup gets rid of being crushed when applying the torsion angle control (Fig.6 (a)), in which the torsion angle of the spring is monitored and fed back. In other words, the torsion spring has a role as a sensor that monitors the pinching force.

\section{Finger Using Double Planetary Gear System}

In parallel with developing the finger mechanism shown in the former section we have been developing an advanced mechanism that covers all of the functions the former mechanism has and has one more DOF; abduction/adduction of the MP joint [10]. It mainly consists of two parts: the double planetary gear system (DPGS) and the compound fourbar linkages as shown in Fig.7. The appearance of the assembled finger, the model developed in 2008, is shown in Fig. 10.

\section{A. DPGS}

Fig.8 describes the structure of the DPGS. It consists of one inner gear in which two sets of a solar gear and three planetary gears are embedded face to face. The left-hand and the righthand solar gears are driven by individual motors (hereafter we call both of them as "motor 1"). The planetary gears located around each solar gear rotate on their own axes and transmit the rotation torque to the inner gear, or take an orbital rotation around the solar gear accompanied by rotating an individual carrier.

\section{B. Abduction/adduction of the MP joint by DPGS}

Abduction/adduction of the MP joint is carried out as follows. A link 2-p (see Fig.9) is connected to the axle of one planetary gear on the carrier. The rotation of the left side and right side solar gears in opposite direction gives rise to the orbital rotation of the planetary gears (the rotation of the carriers), which is also opposite between the left-hand side and the right-hand side. Then the link 2-p of both sides moves forward and backward as shown in the right-hand figure in Fig.9, which induces the abduction/adduction of the MP joint.

\section{Synergic gripping motion}

The new mechanism can achieve the completely identical gripping motion to the former mechanism. Three joints of one finger (DIP, PIP and MP joints) concurrently grip the object if both sides of the solar gears are rotated in the same direction, which provides the identical motion as schematically shown in Fig.2. Fig.11 shows the actual gripping motion of the finger. The sequential view of the left hand side is the case that there is no object to be gripped, in which simultaneous flexion of MP, 


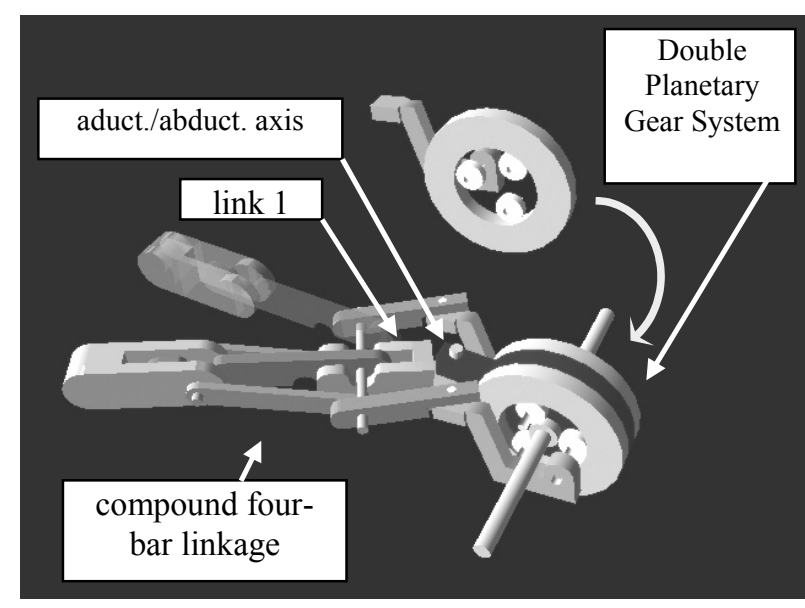

Fig.7 Configuration of the new mechanism

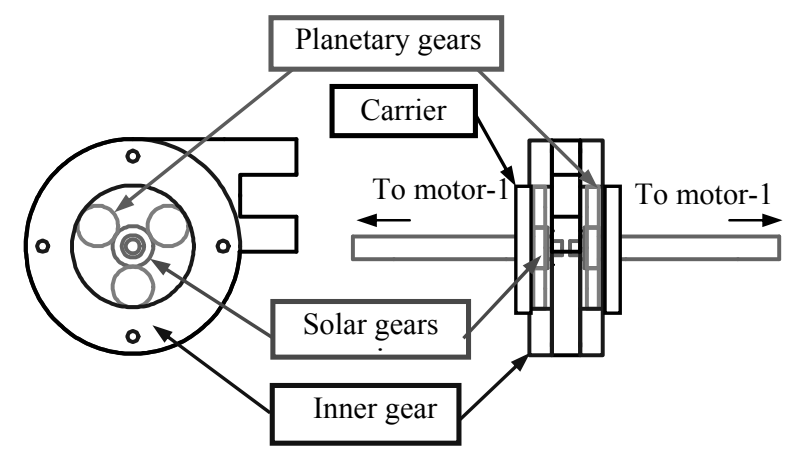

Fig.8 Double planetary gear system (DPGS)

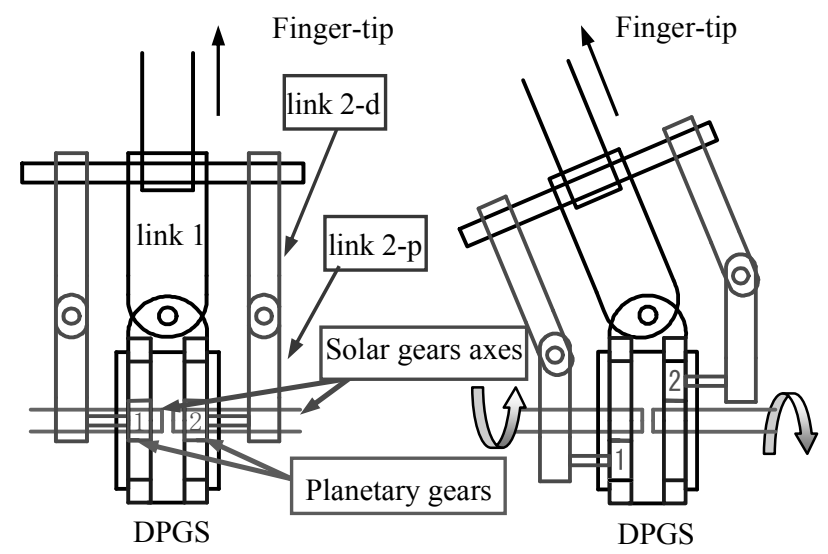

Fig.9 Abduct./adduct. by the DPGS

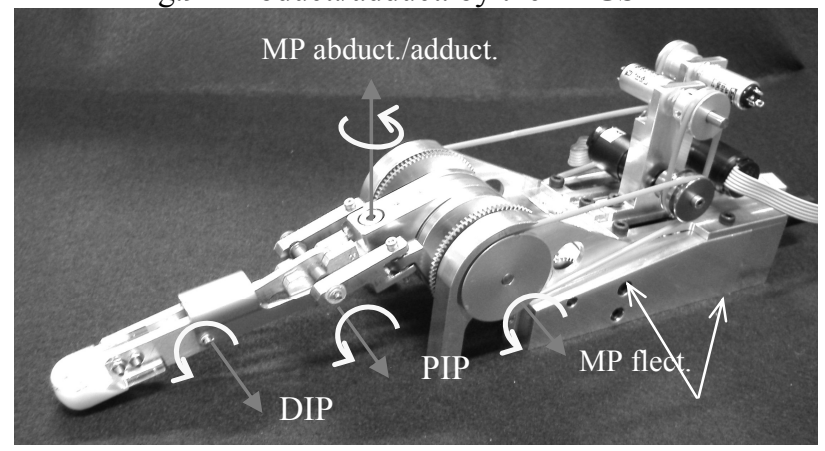

Fig.10 Overview of the finger developed in 2008
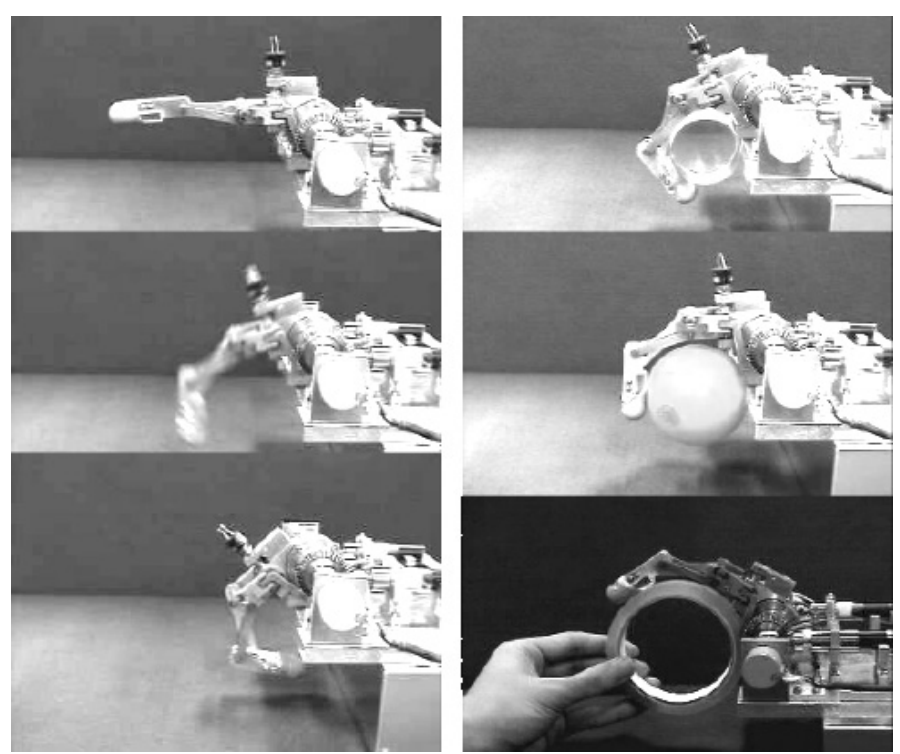

Fig.11 Gripping motion of the finger
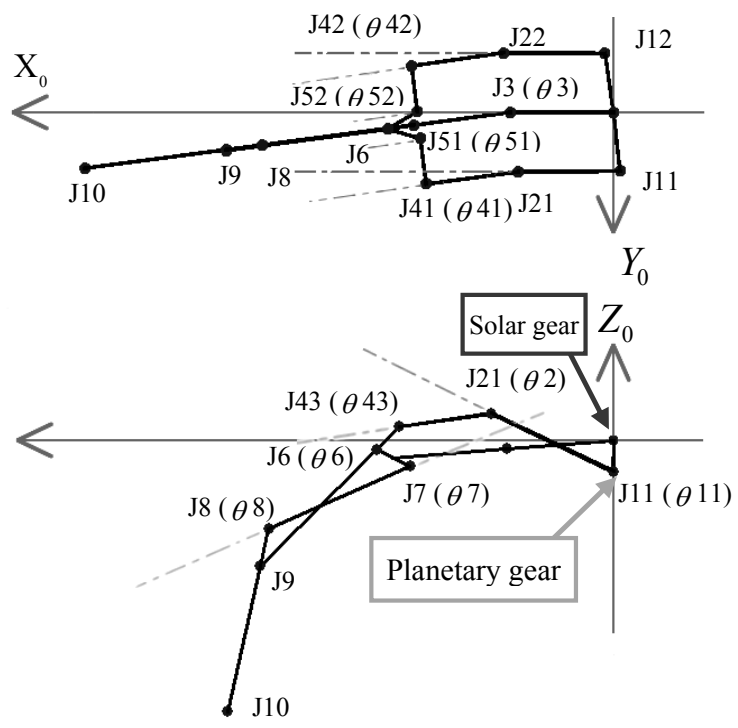

Fig.12 Finger configuration for simulation

PIP and DIP joints occurs by the single rotation of the solar gear. The right hand side shows to grip objects in various size.

It is noteworthy that these gripping motions occur in just one way rotation of the solar gear with no sensory feedback to satisfy the $\mathrm{BD}$, ISD and $\mathrm{SC}$ requirements described in the introduction.

\section{Kinematics and kinetics}

According to the assignment of the joint angles and the links shown in Fig.12, we can derive the kinematics and the kinetics of the finger. In the case of the finger mechanism based on the DPGS we have two solar gears angles $\theta_{S 1}, \theta_{S 2}$, two carrier angles $\theta_{P 1}, \theta_{P 2}$ and one inner gear angle $\theta_{I}$ in the DPGS. First we define two angles as,

$$
\theta_{S D} \equiv\left(\theta_{S 1}-\theta_{S 2}\right) / 2, \quad \theta_{S S} \equiv\left(\theta_{S 1}+\theta_{S 2}\right) / 2
$$


Then we can establish the following relationship of the DPGS angles,

$$
\left[\begin{array}{c}
\Delta \theta_{I} \\
\Delta \theta_{P 1} \\
\Delta \theta_{P 2}
\end{array}\right]=\left[\begin{array}{cc}
-1 / \rho_{S I} & 0 \\
0 & 1 /\left(1+\rho_{S I}\right) \\
0 & -1 /\left(1+\rho_{S I}\right)
\end{array}\right]\left[\begin{array}{l}
\Delta \theta_{S S} \\
\Delta \theta_{S D}
\end{array}\right]=\mathbf{A}\left[\begin{array}{c}
\Delta \theta_{S S} \\
\Delta \theta_{S D}
\end{array}\right]
$$

where, $\rho_{S I}=Z_{I} / Z_{S}$ with $Z_{S}$ and $Z_{I}$ being the number of teeth of the solar gear and the inner gear respectively.

Next we find the following eight kinematical constraints existing in the closed link system,

$$
\begin{gathered}
f_{C 1} \equiv J_{61, x}-J_{62, x}=0, \quad f_{C 2} \equiv J_{61, y}-J_{62, y}=0, \\
f_{C 3} \equiv J_{61, z}-J_{62, z}=0, \\
f_{C 4} \equiv J_{61, x}-J_{63, x}=0, \\
f_{C 5} \equiv J_{61, y}-J_{63, y}=0, \quad f_{C 6} \equiv J_{61, z}-J_{63, z}=0, \\
f_{C 7} \equiv J_{91, x}-J_{92, x}=0, \quad f_{C 8} \equiv J_{91, z}-J_{92, z}=0
\end{gathered}
$$

Partial differentiation with respect to angular variables provides the following equation to derive the infinitesimal variations of

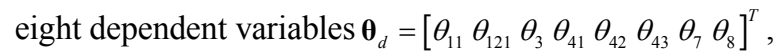

$$
\Delta \boldsymbol{\theta}_{d}=-\mathbf{B}^{-1} \mathbf{C A}\left[\begin{array}{ll}
\Delta \theta_{S S} & \Delta \theta_{S D}
\end{array}\right]^{T}
$$

where, $\mathbf{B}=\partial \mathbf{f}_{C} / \partial \boldsymbol{\theta}_{d} \in \mathfrak{R}^{8 \times 8}$ and $\mathbf{C}=\partial \mathbf{f}_{C} / \partial \boldsymbol{\theta}_{\text {ind }} \in \mathfrak{R}^{8 \times 3}$ with $\mathbf{f}_{C}=\left\{f_{C i} \mid i=1, \cdots, 8\right\}$ and $\boldsymbol{\theta}_{\text {ind }}=\left[\theta_{I} \theta_{P 1} \theta_{P 2}\right]^{T}$.

At the same time the infinitesimal variation of the finger tip position $\mathbf{J}_{10}=\left[\begin{array}{lll}J_{10 x} & J_{10 y} & J_{10 z}\end{array}\right]^{T}$ will be,

$$
\Delta \mathbf{J}_{10}=\left(\mathbf{E}-\mathbf{D B}^{-1} \mathbf{C}\right) \mathbf{A}\left[\begin{array}{ll}
\Delta \theta_{S S} & \Delta \theta_{S D}
\end{array}\right]^{T}=\mathbf{\Lambda}\left[\begin{array}{ll}
\Delta \theta_{S S} & \Delta \theta_{S D}
\end{array}\right]^{T}
$$

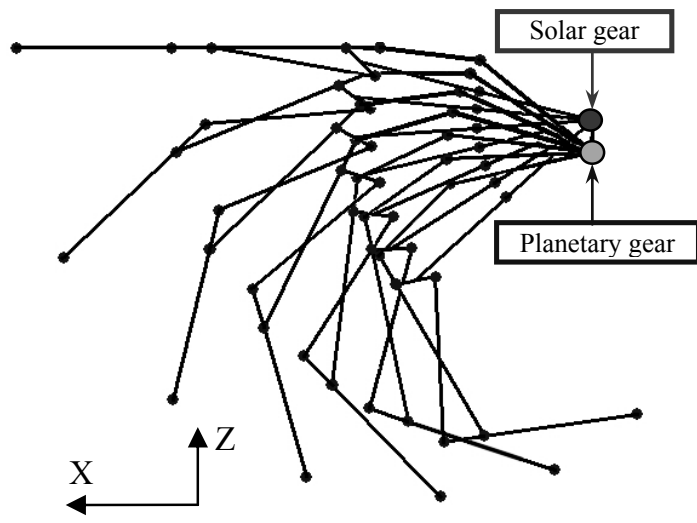

(a) Finger motion when two solar gears rotate in the same direction

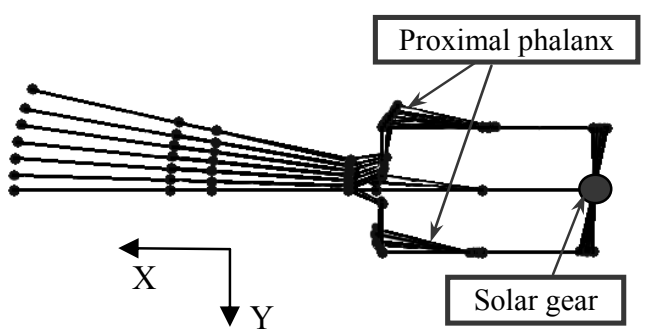

(b) Finger motion when two solar gears rotate in the opposite direction

Fig.13 Kinematical simulation of the finger motion

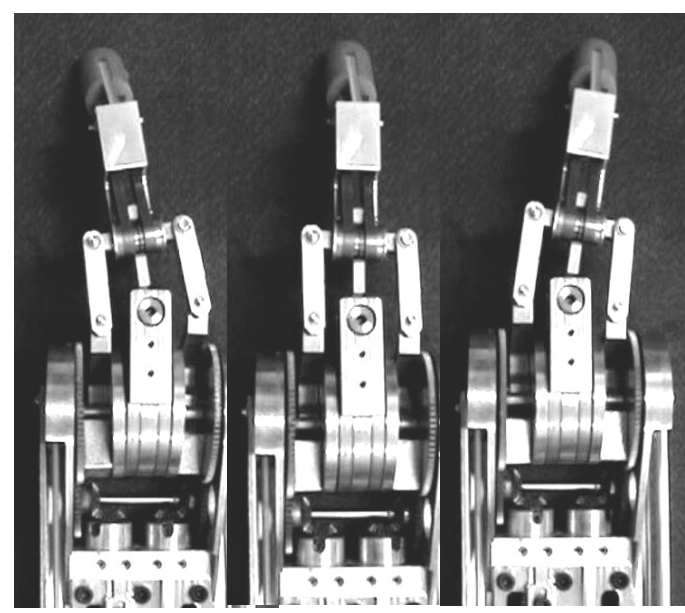

Fig.14 The abduct./adduct. motion of the finger
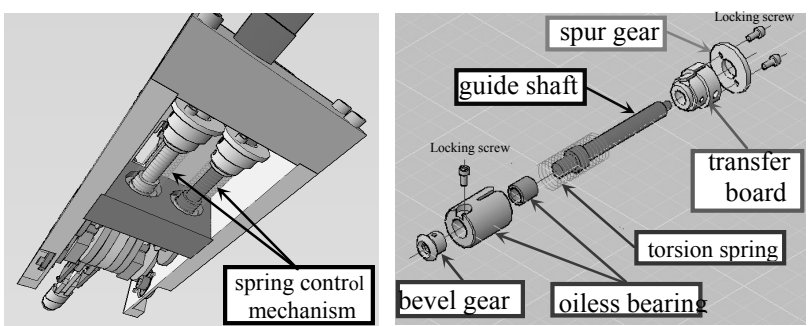

Underside view of the base

Spring control mechanism

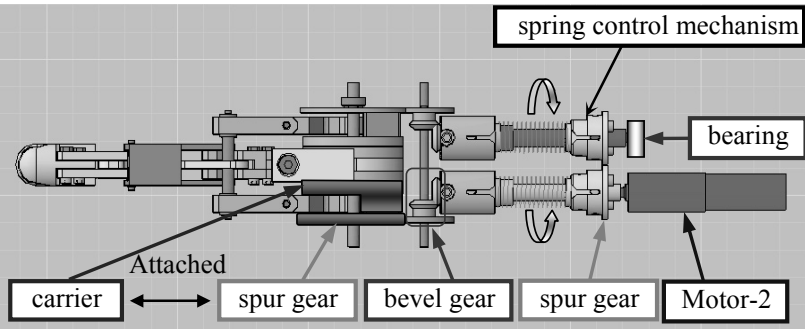

Fig. 15 The parts of the spring controller

with $\mathbf{D}=\partial \mathbf{J}_{10} / \partial \boldsymbol{\theta}_{d} \in \mathfrak{R}^{3 \times 8} \quad, \quad \mathbf{E}=\partial \mathbf{J}_{10} / \partial \boldsymbol{\theta}_{\text {ind }} \in \mathfrak{R}^{3 \times 3}$ and the Jacobian $\boldsymbol{\Lambda} \equiv\left(\mathbf{E}-\mathbf{D B}^{-1} \mathbf{C}\right) \mathbf{A}$.

Fig. 13 shows the kinematical motion of the finger. Fig.13 (a) is the result when two solar gears rotate in the same direction and Fig.13 (b) is those for the opposite direction. Both results are assumed that the carriers do not rotate. As shown, the solar gears' rotation in the same direction gives rise to the synergetic flexion of MP, PIP and DIP joints. On the other hand their rotation in the opposite direction gives rise to the abduction/adduction of the MP joint about $\pm 25^{\circ}$ that is almost the same range as those of a human finger. Fig. 14 shows the actual abduction/adduction of the machine.

\section{E. Pinching force control}

It also needs springs for the finger to allow a back-drivable motion that is identical to the finger based on the PGS. Fig. 15 shows the assembling view of the spring controller. We need two torsion springs for the carriers of both sides of DPGS. But 


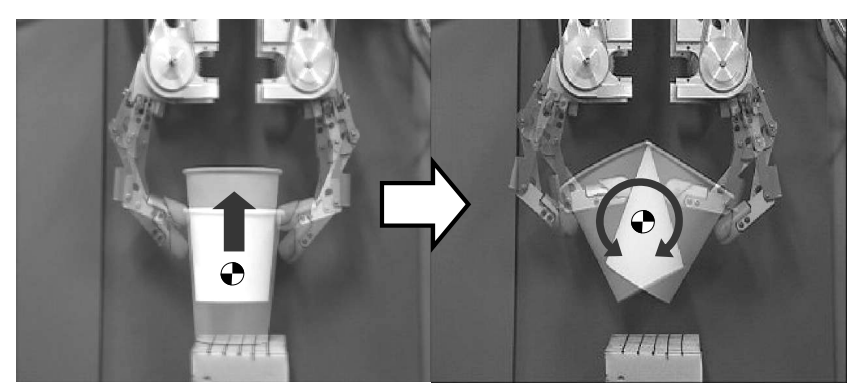

(a) Lifting and rolling an object

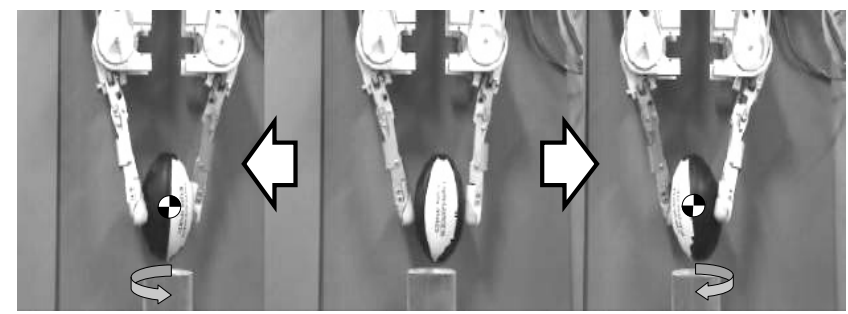

(b) Yawing an object

Fig.16 Object handling by two fingers

the developed spring controller allows for one motor (Motor 2) to twist both of the torsion springs simultaneously.

\section{$F$. Pinching an object by two fingers}

The mechanism based on the DPGS allows two fingers to handle the object with simple control schemes as same as the mechanism based on the PGS shown in the former section. Fig.16 (a) is the example for lifting and rolling the paper cup. In this motion two solar gears in one finger rotates identically and the torsion springs that connect to the left-hand and righthand carriers of the DPGS are controlled by the motor 2 to keep the constant torsion angle of the spring. Therefore this motion can be achieved by the mechanism based on the PGS, but the motion shown in Fig.16 (b) can not be. It is an object yawing, which can be achieved by fingers having a DOF of abduction/ adduction. During the motion two solar gears rotates in the opposite direction.

\section{CONCLUSIONS}

This paper shows two finger mechanisms; one is based on the planetary gear system (PGS) and the other is based on the originally-devised double planetary gear system (DPGS). Both mechanisms will satisfy the requirements of BD (backdrivability), ISD (inherently safe design) and SC (simple control), all of which are indispensable properties for robot hand or prosthesis. The finger with PGS will also satisfy the AOD (all-in-one design) for developing a multiple finger hand. Moreover the mechanisms have the following properties;

(a) Its kinematics, kinetics and dynamics can be solved in reliable level because they have no tendon wire.

(b) Although they have no sensors in their finger portion for the sake of ISD, the spring equipped in the PGS or DPGS has a role of force sensor.

The above facts will enhance to use the mechanisms into applications that require more precise and dexterous works.

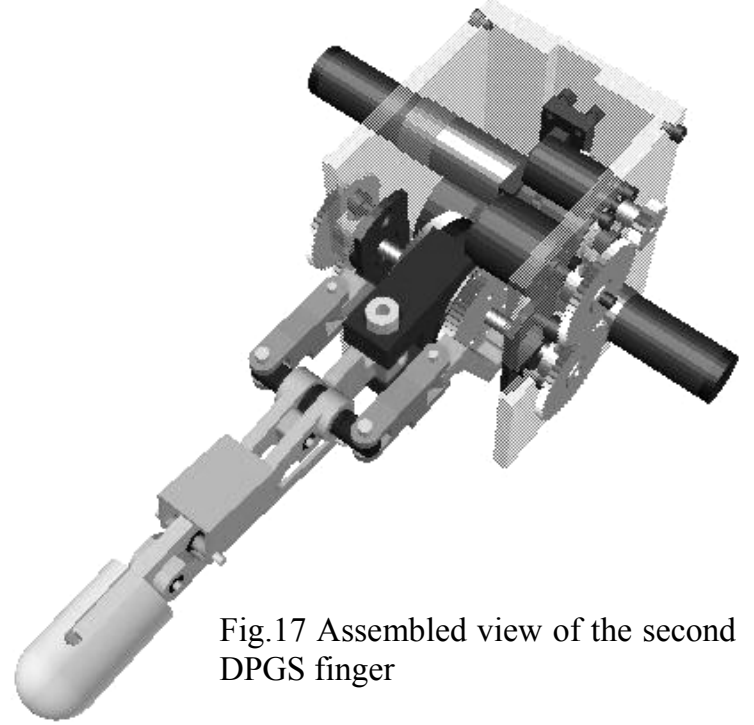

We are now developing the finger based on the DPGS with a new reduced volume design in the prime mover part as shown in Fig. 17 in order to compose a hand with multiple fingers that will satisfy the AOD (all-in-one design).

\section{ACKNOWLEDGMENT}

The authors thank the Japan Society for the Promotion of Science, via Grants-in-Aid for Scientific Research: No. 20560242 'Development of Artificial Hand using the Double Planetary Gear System and the Handling of Objects', for their support in the pursuance of this work.

\section{REFERENCES}

[1] Kawasaki, H., et. al., Mechanism of Anthropomorphic Robot Hand: Gifu Hand I, J. Robot. And Mech., 11,4, pp.269-273, 1999.

[2] Namiki, A., Y. Imai, M. Kaneko and M. Ishikawa, Development of a Highspeed Multifingered Hand System, Proc. of Int. Conf. On Intelligent Manipulation and Grasping, Genova-Itary, pp.85-90, 2004.

[3] Salisbury, K. and B. Roth, Kinematics and force analysis of articulate mechanical hands, ASME J. Mechanism, Transmissions and Autom. In Design, Vol.105, pp.35-41, 1983

[4] Maekawa, H., et al., Development of Three-Fingered Robot Hand with Stiffness Control Capability, Mechanism, Vol.2, No.5, pp.483-494, 1992.

[5] Lovchik, C.S. and M.A. Diftler, The Robonaut Hand:A Dextrous Robot Hand for Space, Proc. of the 1999 IEEE Int. Conf. On Robotics and Autom, pp.907-912, 1999.

[6] Lee, Y-T, H-R Choi, W-K Chung and Y. Youm, Stiffness Control of a Coupled Tendon-Driven Robot Hand, IEEE Control Systems, pp.10-19, 1994.

[7] Harada Electric Industry Co., Myo-electric controlled forearm prosthesis SH-1, URL http://www.h-e-i.co.jp.

[8] Koganezawa, K., Artificial Finger with Shape-Fitting Mechanism, Proceedings of the International Conference on Intelligent Manipulation and Grasping, pp. 103-109, Genova, Italy, 2004

[9] Martin, E. and T. L. C. Gosselin, SARAH Hand Used for Space Operations on STVF Robot, Proceedings of the International Conference on Intelligent Manipulation and Grasping, pp. 279-284, Genova, Italy, 2004

[10] Koganezawa, K. and Y. Ishizuka, Novel Mechanism of Artificial Finger using Double Planetary Gear System, Proceedings of the 2008 IEEE/RSJ International Conference on Intelligent Robots and Systems, pp. 3184-3191, Nice, France, Sept 22-26, 2008. 